PRINTED IN GREAT BRITAIN BY W. LEWIS, M.A., AT THE UNIVERSTTY PRESS, CAMBRIDGE 


\section{Proceedings of the CAMBRIDGE PHILOSOPHICAL SOCIETY}




\section{CAMBRIDGE \\ UNIVERSITY PRESS \\ LONDON: BENTLEY HOUSE \\ NEW YORK, TORONTO, BOMBAY \\ CALCUTTA, MADRAS: MACMILLAN \\ TOKYO: MARUZEN COMPANY IJTD \\ All rights reserved}




\section{Proceedings of the}

\section{CAMBRIDGE PHILOSOPHICAL SOCIETY}

VOLUME 34

CAMBRIDGE

AT THE UNIVERSITY PRESS

1938 
PRINTED IN GREAT BRITAIN 


\section{O N T E N T S}

Allen, J. F. and Misener, A. D. The surface tension of liquid helium $\quad$ - $\quad 299$ and Shire, E. S. Thermal equilibrium at temperatures below $1^{\circ}$ absolute $\quad 301$

Babbage, D. W. The resolution of monoidal Cremona transformations of threedimensional space

Banerji, D. P. The expansion of an arbitrary function in a series of conal or toroidal functions

Bartlett, M. S. Further aspects of the theory of multiple regression . . . . 33

Bennett, W. E. The internal conversion electrons of actinon _ . . . $\quad$ - $\quad 282$

Bosworth, R. C. L. The evaporation of concentrated films of sodium . $\quad$ - $\quad$ - $\quad 262$

Bower, J. C. Variation of ionization with range of $\alpha$-particles, protons, deuterons and ${ }^{3} \mathrm{H}$ particles

, Bretscher, E. and Gilbert, C. W. The disintegration of boron by slow

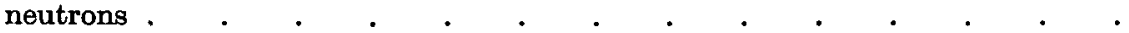

Bretscher, E., Gilbert, C. W. and Bower, J. C. The disintegration of boron by slow neutrons .

Budden, K. G. An apparatus for recording the strength of wireless signals from a Morse sender .

Cernuschi, F. Contributions to the statistical theory of adsorption. I. The elementary theory of adsorption in a first and second layer

Chang, T. S. Superlattice formation of the type $A B$ in alloys with applications to the theory of adsorption.

Corben, H. C. The straggling of electrons due to radiation and ionization loss

Coulson, C. A. Self-consistent field for molecular hydrogen .

Daniels, H. E. The effect of departures from ideal conditions other than non-

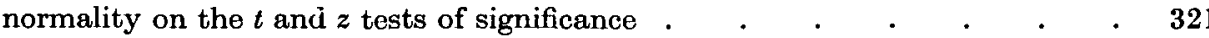

Davenport, $\mathbf{H}$. Note on an identity connected with diophantine approximation .

Ditchburn, R. W. and Roulston, K. I. The sputtering of oxide-covered magnesium surfaces

Dube, G. P. The electrical resistance of a metal at low temperatures and Matthiessen's rule.

- Contributions to the statistical theory of adsorption. II. The theory of twolayer adsorption with correlation in the first layer .

Dunworth, J. V. and Feather, N. Note on the production of positron-electron pairs during the passing of $\beta$-particles through matter

Absorption and coincidence experiments on the radiations from the radioactive sodium, $\mathrm{Na}^{24}$.

Du Val, $\mathbf{P}$. The fixed part of the canonical system on an algebraic surface . . 1

Easterfield, T. E. An extension of a theorem of Kulakoff . . . . . . . $\quad 316$ 
Easthope, C. E. The critical ordering temperature in alloys. II. The existence of a two-phase region

Edge, W. L. Determinantal representations of $x^{4}+y^{4}+z^{4} \quad . \quad$. . . . . 6

Erdélyi, A. The Hankel transform of Whittaker's function $W_{k, m}(z) \quad \cdot \quad \cdot \quad \cdot \quad 28$

Feather, N. Note on the absorption limits for the primary $\beta$-particles of mesothorium 2 and uranium $X_{2}$

Further possibilities for the absorption method of investigating the primary $\beta$-particles from radioactive substances

and Dunworth, J. V. Note on the production of positron-electron pairs during the passing of $\beta$-particles through matter . . . . . . .

and Dunworth, J. V. Absorption and coincidence experiments on the radiations from the radioactive sodium, $\mathrm{Na}^{24}$.

Fejér, L. Zur Summabilitätstheorie der Fourierschen und Laplaceschen Reihe

Fowler, R. H. A modernized version of Gibbs' use of the grand canonical ensemble and Jones, $\mathbf{H}$. The properties of a perfect Einstein-Bose gas at low temperatures .

Fuchs, $\mathbf{x}$. The conductivity of thin metallic films according to the electron theory of metals .

Fuchs, W. H. J. and Weiss, P. Uniqueness theorems and the maximumminimum principle for a type of non-linear partial differential equations .

Gilbert, G. W., Bower, J. C. and Bretscher, E. The disintegration of boron by slow neutrons

Gillis, J. Corrigendum .

Goldstein, S. On the velocity and temperature distributions in the turbulent wake behind a heated body of revolution.

Note on the velocity and temperature distributions behind a heated body of revolution

Green, A. E. The moment of the fluid forces acting on a plate which is gliding on the surface of a stream

The mean value of the fluctuations in pressure and pressure gradient in a turbulent fluid.

Hall, A. A. and Hislop, G. S. Velocity and temperature distributions in the turbulent wake behind a heated body of revolution

Hardy, G. H. A further note on Ramanujan's arithmetical function $\mathbf{T}(n)$. .

Hartree, D. R. and Hartree, W. Wave functions for negative ions of sodium and potassium

Hartree, W. and Hartree, D. R. Wave functions for negative ions of sodium and poteissium

Heilbronn, H. On Euclid's algorithm in real quadratic fields . . . . . $\quad$. $\quad 521$

Hirschlaff, E. The velocity of sound in liquid nitrogen _ . . . . . . 296 
Hislop, G. S. and Hall, A. A. Velocity and temperature distributions in the turbulent wake behind a heated body of revolution

Howarth, L. Concerning the velocity and temperature distributions in plane and axially symmetrical jets. Concerning secondary flow in straight pipes . . . . . . . . . $\quad$. 335

Jánossy, L. The penetrating power of cosmic-ray shower particles

Jeffreys, H. The use of minimum $\chi^{2}$ as an approximation to the method of maximum likelihood

Johnston, J. E. and Skinner, H. W. B. Soft X-ray bands from dilute alloys .

Jones, H. A discussion of the thermal expansion and conductivity in liquid helium II and Fowler, R. H. The properties of a perfect Einstein-Bose gas at low temperatures

Kara-Michailova, E. The total energy of the $\gamma$-radiation emitted from the active deposit of actinium .

Kemmer, $\mathbf{N}$. The charge-dependence of nuclear forces

Makinson, R. E. B. The thermal conductivity of metals

Massey, H. S. W. and Mohr, C. B. O. Anomalous scattering of $\alpha$-particles and long-range nuclear forces .

Michailova, E. Kara-, see Kara-Michailova, E.

Misener, A. D. The "overshoot phenomenon" in superconductivity .

- and Allen, J. F. The surface tension of liquid helium .

Mohr, C. B. O. and Massey, H. S. W. Anomalous scattering of $\alpha$-particles and long-range nuclear forces .

Monroe, E. On the energies associated with the two-centre problem. I. General features of the energy surface .

Mott, N. F. Note on the contact between a metal and an insulator or semiconductor

Moullin, E. B. A note on the melting point of paraffins and fatty acids

Nagendra Nath, N. S., see Nath, N. S. N.

Nath, N. S. N. The diffraction of light by supersonic waves in solids . . . 213

Pitt, H. R. Mercerian theorems

Roberts, J. K. Some properties of mobile and immobile adsorbed films $\quad$ - $\quad$ - $\quad 399$

- The theory of adsorption when each particle occupies more than one site $\quad . \quad 577$

Roulston, K. I. and Ditchburn, R. W. The sputtering of oxide-covered magnesium surfaces

Rowland, E. N. Note on fluctuations and the shot effect

Rushbrooke, G. S. A note on an assumption in the theory of cooperative phenomena 
Segal, I. E. Fiducial distribution of several parameters with application to a normal system

Shire, E. S. and Allen, J. F. Thermal equilibrium at temperatures below $1^{\circ}$ absolute

Skinner, H. W. B. and Johnston, J. E. Soft X-ray bands from dilute alloys .

Sosnowski, L. and Westcott, C. H. Further experiments on the slow neutrons transmitted by cadmium: influence of the temperature of the slowing down medium .

Stansfield, R. G. A search for ionization of hydrogen by diffusion through palladium

Suitability of a diamagnetic crystal for the measurement of magnetic fields .

Tibbs, S. R. Electronic energy bands in metallic copper and silver

Todd, J. A. Birational transformations possessing fundamental curves

Turan, P. Über die monotone Konvergenz der Cesàro-Mittel bei Fourier- und Potenzreihen

Val, P. du, see Du Val, P.

Viktorin, O. Production of the long-lived radioactive cobalt isotope from nickel bombarded by fast neutrons

Wang, J. S. Statistical theory of adsorption with long-range interaction

- The kinetics of adsorption with long-range interaction between adsorbed particles

Weiss, P. and Fuchs, W. H. J. Uniqueness theorems and the maximum-minimum principle for a type of non-linear partial differential equations . . .

Westcott, C. H. and Sosnowski, L. Further experiments on the slow neutrons transmitted by cadmium: influence of the temperature of the slowing down medium

Whittaker, J. M. The shot effect with space charge

Wilson, A. H. Lattice changes associated with the formation of superlattices in alloys

_- The binding energies of the hydrogen isotopes . $\quad . \quad . \quad . \quad . \quad$.

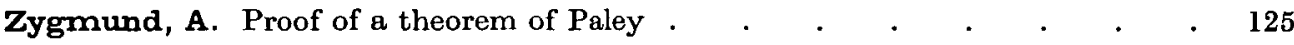

Proceedings . . . . . . . . . . . . . . . . . 635

Balance Sheet .

\section{PLATES}

Plate I. To illustrate Mr Bennett's paper

facing 284

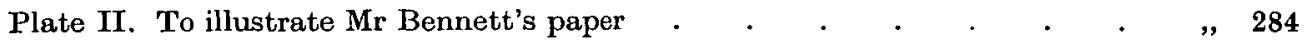

Plate III. To illustrate Messrs Shire and Allen's paper . . . . . . . " 304 


\title{
Now Ready
}

The second volume in the new series

THE CAMBRIDGE LIBRARY OF MODERN SCIENCE

\section{Background to Modern Science}

\author{
Edited by J. NEEDHAM \& W. PAGEL. 7s. 6d. net
}

Here, in one volume, is a complete survey of the development of science in the last forty years, each section being the work of an expert: Professor F. M. Cornford, Sir William Dampier, the late Lord Rutherford, Professor W. L. Bragg, Dr F. W. Aston, Sir Arthur Eddington, Professor J. A. Ryle, the late Professor G. H. F. Nuttall, Professor R. C. Punnett, and Professor J. B. S. Haldane.

The first volume in this series, The Evolution of Physics by EINSTEIN \&? $I \mathcal{N F E L D}$, published at $8 s .6 d$. net in the spring, has met with an immediate success here and in America.

\section{Stellar Dynamics}

\author{
By W. M. SMART. 30s. net
}

This book presents the subject in its full mathematical and observational development. Contents: Introduction; A Single Star-Drift; The Solar Motion; The Two Star-Streams; The Ellipsoidal Theory; Statistical Parallaxes derived from Stellar Motions; The Space Distribution of the Stars derived from their Proper Motions; General Theorems of Stellar Statistics; Star Clusters; The Dynamics of Stellar Systems; Galactic Rotation; The Dynamics of the Galaxy; Appendix.

\section{Cheap Edition}

\section{Sir Arthur Eddington NEW PATHWAYS IN SCIENCE \\ 5s. net}

"A fine book which no scientifically minded man or woman can neglect." WALTER G. BELL in the Daily Telegraph

\section{CAMBRIDGE UNIVERSITY PRESS}




\section{O N T E N T S}

FeJÉr, Leopold. Zur summabilitätstheorie der Fouriersehen und Laplaceschen Reihe . . . . . . . . . . . . 503

Piтt, H. R. Mercerian theorems . . . . . . . . 510

HeItbrons, H. On Euclid's algorithm in real quadratic fields . . . 521

Fuchs, W. H. J. and Weiss, P. Uniqueness theorems and the maximumminimum principle for a type of non-linear partial differential equations .

GreEn, A. E. The mean value of the fluctuations in pressure and pressure gradient in a turbulent fluid .

Conber, H. C. The straggling of electrons due to radiation and ionization loss. (With 3 Figures in the Text) . . . . . . . .

Hartree, D. R. and Hartree, W. Wave functions for negative ions of sodium and potassium .

Dube, G. P. The electrical resistance of a metal at low temperatures and Matthiessen's rule.

Мотт, N. F. Note on the contact between a metal and an insulator or semiconductor. (With 1 Figure in the Text) . . . . . . .

Fowler, R. H. and Jones, H. The properties of a perfect Einstein-Bose gas at low temperatures

RoBerts, J. K. The theory of adsorption when each particle occupies more than one site. (With 5 Figures in the Text) . . . . . .

Dube, G. P. Contributions to the statistical theory of adsorption. II. The theory of two-layer adsorption with correlation in the first layer. (With 4 Figures in the Text)

FEATHER, N. Further possibilities for the absorption method of investigating the primary $\beta$-particles from radioactive substances. (With 7 Figures in the Text)

Viktorin, Otakar. Production of the long-lived radioactive cobalt isotope from nickel bombarded by fast neutrons

JÁnossy, L. The penetrating power of cosmic-ray shower particles. (With 2 Figures in the Text)

Ditchidrn, R. W. and Roulston, K. I. The sputtering of oxide-covered magnesium surfaces. (With 1 Figure in the Text) . . . . .

Stansfield, R. G. Suitability of a diamagnetic crystal for the measurement of magnetic fields. (With 4 Figures in the Text)

Proceedings

Balance Sheet

LONDON: CAMBRIDGE UNIVERSITY PRESS

INDIA: MACMILLAN

TOKYO: MARUZEN COMPANY LIMITED

Dutch Photo Reprint 